\title{
Proprotein Convertase Subtilisin/Kexin Type 9 in the Inflammation-Related Dyslipidemia of Patients with Axial Spondyloarthritis is Related to Disease Activity.
}

\section{Laura de Armas-Rillo}

Universidad Europea de Canarias

Juan Carlos Quevedo-Abeledo

Hospital Universitario de Gran Canaria Dr Negrin

Antonia de Vera-González

Hospital Universitario de Canarias

Alejandra González-Delgado

Hospital Universitario de Canarias

José A. García-Dopico

Hospital Universitario de Canarias

Alejandro Jimenez-Sosa

Hospital Universitario de Canarias

Carlos Rodríguez-Lozano

Hospital Universitario de Gran Canaria Dr Negrin

Miguel A. González-Gay

Hospital Universitario Marques de Valdecilla

Iván Ferraz-Amaro ( $\square$ iferrazamaro@hotmail.com )

Hospital Universitario de Canarias https://orcid.org/0000-0003-0197-5267

Research article

Keywords: Proprotein, convertase, subtilisin/kexin type 9 (PCSK9), protease

Posted Date: April 7th, 2020

DOl: https://doi.org/10.21203/rs.3.rs-21063/v1

License: (1) This work is licensed under a Creative Commons Attribution 4.0 International License.

Read Full License 


\section{Abstract}

Introduction: Proprotein convertase subtilisin/kexin type 9 (PCSK9) is a serine protease that regulates cholesterol metabolism through low-density lipoprotein receptor degradation and that has been linked to cardiovascular risk. The purpose of the present study was to examine whether PCSK9 levels are related to both abnormalities in the lipid profile and the development of severe atherosclerosis that often occurs in patients with axial spondyloarthritis (axSpA).

Methods: Cross-sectional study that encompassed 545 individuals; 299 patients with axSpA and 246 statin use-matched controls. PCSK9, lipoproteins serum concentrations and standard lipid profiles were analyzed in patients and controls. Carotid intima-media thickness (cIMT) and carotid plaques were assessed in patients. A multivariable analysis, adjusted for standard cardiovascular risk factors, was performed to evaluate the influence of PCSK9 on axSpA related dyslipidemia and subclinical carotid atherosclerosis.

Results: Most lipid panel parameters (total cholesterol, HDL- and LDL-cholesterol, lipoprotein (a) and apoliprotein A1) were significantly lower in axSpA patients than controls. PCSK9 serum levels (beta coef. $-44[95 \% \mathrm{Cl}-60--27] \mathrm{ng} / \mathrm{dl}, \mathrm{p}=0.000$ ) were also downregulated in axSpA patients after fully multivariate adjustment, including other lipid profile-related molecules modifications that the disease generates. Unadjusted higher levels of PCSK9 were associated with carotid plaque in axSpA patients (beta coef. 27 [95\% Cl 9-45], $p=0.003$ ). ASDAS-CRP was found to be independently and significantly related to PCSK9 (beta coef. 10 [95\% Cl 1-18] ng/dl, p=0.023) after analyzing fully adjusted models that took age, sex, and the rest of lipid profile molecules into account. Whereas patients taking prednisone showed higher serum levels of PCSK9 $(55$ [95\% Cl 24-8]) ng/ml, $\mathrm{p}=0.001)$, those under anti-TNF alpha therapies exhibited lower levels (beta coef. -26 [95\% Cl -43- -9], $p=0.003$ ).

Conclusion: PCSK9 is downregulated in patients with axSpA. Disease activity is positive and significantly related to PSCK9. Anti-TNF-therapy yields reduction in PCSK9 serum levels.

\section{Introduction}

Axial spondyloarthritis (axSpA) encompasses a group of inflammatory disorders that share a number of clinical features including inflammation of axial joints, asymmetric oligoarthritis, dactylitis, and enthesitis. Patients with axSpA are prone to an increased and premature prevalence of atherosclerosis (1, 2). This is due to the compound effects of a genetic component, classic cardiovascular (CV) risk factors, inflammation, disease severity and the therapy used to manage the disease (3). In the ASAS-COMOSPA study (4), an observational, cross-sectional, multicenter and international study involving more than 3,000 subjects with axSpA that sought to assess the prevalence of comorbidities, one of the most frequent comorbidities was hypercholesterolemia, which was present in $27 \%$ of the patients. In this regard, an increased risk of hyperlipidemia (prevalence ratio 1.2 [95\% $\mathrm{Cl} 1.1-1.3]$ ) was found in patients with axSpA compared to controls (5). Although the role of inflammation in atherogenesis and plaque 
formation in axSpA is not well understood, inflammation in axSpA has been linked to an atherogenic lipid profile in these individuals (6-8). This disrupted lipid molecules pattern may be responsible for the subsequent atherosclerosis burden that these patients exhibit (7).

Proprotein convertase subtilisin kexin 9 (PCSK9), a serine protease, plays an important role in low-density lipoprotein (LDL) metabolism. PCSK9, which is synthesized primarily in the liver, enters the circulation, where it binds to hepatic LDL receptors and targets them for degradation (9). This process reduces the capacity of the liver to bind and remove LDL-cholesterol, resulting in increased LDL-cholesterol levels (10). Consistent evidence from pre-clinical studies indicates that modulation of PCSK9 activity may have potential positive effects on coronary heart disease $(11,12)$. The reduced incidence of cardiovascular events in patients bearing PCSK9 loss-of-function mutations supported this concept and provided a strong rationale for the development of molecules capable of inhibiting PCSK9 function (13). Blockading the interaction between PCSK9 and LDL receptors by the use of a fully human monoclonal antibody that binds PCSK9 leads to lower LDL-cholesterol levels in patients with hypercholesterolemia (14), reducing the rate of cardiovascular events $(15,16)$. Interestingly, PCSK9 has been found to be downregulated in patients with rheumatoid arthritis (17).

To the best of our knowledge, there is no information on the role of PCSK9 in the inflammation-related dyslipidemia of patients with axSpA. For this reason, we conducted a study to determine whether PCSK9 is associated with the changes that inflammation and the disease exert over the lipid patterns of axSpA patients. We additionally aimed to establish if PCSK9 is linked to atherosclerosis based on carotid ultrasound assessments in individuals with axSpA.

\section{Materials And Methods}

\section{Study participants}

This was a cross-sectional study that included 299 patients with axSpA and 246 controls. All of them were 18 years old or older. axSpA patients included in this study fulfilled the Assessment of SpondyloArthritis International Society (ASAS) classification criteria (18). They were diagnosed by rheumatologists and periodically followed-up at rheumatology outpatient clinics. For the purpose of inclusion in the present study, axSpA disease duration needed to be $\geq 1$ year. axSpA patients undergoing biologic therapy (anti-TNF alpha therapies) were not excluded from the present study. Likewise, since glucocorticoids may be used in the management of axSpA, patients taking prednisone, or an equivalent dose $\leq 10 \mathrm{mg} /$ day, were not excluded. Controls included in the current study were subjects without any known condition or drug treatment history that could influence lipids and who were not taking any lipidlowering medications other than statins. None of the controls was receiving glucocorticoids. However, patients were excluded if they had a history of cancer or any other chronic disease, evidence of active infection or a glomerular filtration rate $<60 \mathrm{ml} / \mathrm{min} / 1.73 \mathrm{~m}^{2}$. The study protocol was approved by the Institutional Review Committee at Hospital Universitario de Canarias and Hospital Doctor Negrín, both in 
Spain, and all subjects provided informed written consent (Approval Number 2016/64). Research carried out with human subjects was in compliance with the Helsinki Declaration.

\section{Data collection}

Surveys of axSpA patients were performed to assess cardiovascular risk factors and medication. Subjects completed a questionnaire and underwent a physical examination to determine anthropometric measurements and blood pressure. Medical records were reviewed to ascertain specific diagnoses and medications. Hypertension was defined as a systolic or a diastolic blood pressure higher than, respectively, 140 and $90 \mathrm{mmHg}$. The atherogenic index was calculated using the total cholesterol/HDL cholesterol ratio. Two clinical indexes of disease activity (Bath Ankylosing Spondylitis Disease Activity Index [BASDAl] and Ankylosing Spondylitis Disease Activity Score [ASDAS]) $(19,20)$, a functional status index (Bath Ankylosing Spondylitis Functional Index [BASFI]) (21), a metrological index (Bath Ankylosing Spondylitis Metrology Index [BASMI]) (22), and an enthesitis index (Maastricht Ankylosing Spondylitis Enthesitis Score [MASES]) (23) were assessed in all patients at the time of their evaluation visit.

\section{Lipids and PCSK9 assessments}

Fasting serum samples were collected and frozen at $-80^{\circ} \mathrm{C}$ until analysis of circulating lipids. Human PCSK9 was measured using an ELISA kit (R\&D Duoset). Intra- and inter-assay coefficients of variation were $<5 \%$ and $6.3 \%$, respectively. Cholesterol, triglycerides, and high-density lipoprotein (HDL)-cholesterol were measured using the enzymatic colorimetric assay (Roche). Cholesterol ranged from 0.08 to $20.7 \mathrm{mmol} / \mathrm{I}$ (intra-assay coefficient of variation $0.3 \%$ ); triglycerides ranged from 4 to $1.000 \mathrm{mg} / \mathrm{dl}$ (intraassay coefficient of variation 1.8\%); and HDL-cholesterol ranged from 3 to $120 \mathrm{mg} / \mathrm{dl}$ (intra-assay variation coefficient $0.9 \%$ ). LDL-cholesterol was calculated using the Friedewald formula. Additionally, standard techniques were used to measure plasma glucose, C-reactive protein (CRP), and serum lipids.

\section{Carotid ultrasound assessment}

Carotid ultrasound was performed to determine carotid intima-media thickness (cIMT) in the common carotid artery and to detect focal plaques in the extracranial carotid tree both in patients with axSpA and in controls $(24,25)$. A commercially available scanner, Mylab 70, Esaote (Genoa, Italy) equipped with a 7$12 \mathrm{MHz}$ linear transducer and an automated software-guided radiofrequency technique - Quality Intima Media Thickness in real-time (QIMT, Esaote, Maastricht, Holland) - was used for this purpose. Based on the Mannheim consensus, plaque criteria in the accessible extracranial carotid tree (common carotid artery, bulb and internal carotid artery) were defined as follows: a focal protrusion in the lumen measuring at least cIMT > $1.5 \mathrm{~mm}$; a protrusion at least $50 \%$ greater than the surrounding clMT; or an arterial lumen encroaching $>0.5 \mathrm{~mm}(26)$.

\section{Statistical analysis}

Demographic and clinical characteristics in patients with axSpA and controls were described as mean \pm standard deviation or percentages for categorical variables. For non-normally distributed continuous variables, data were expressed as a median and interquartile range (IQR). Univariate differences between 
patients and controls were assessed through T Student, U Mann-Whitney, Chi squared or Fisher Exact tests according to normal distribution or number of subjects. Differences between patients and controls regarding their lipid profiles was assessed through multivariable regression analysis. Confounding variables in this analysis were those with a statistical ' $p$ ' value lower than 0.20 for those differences in traditional $\mathrm{CV}$ risk factors between patients and controls. To neutralize the effect of other modifications on the lipid profile, an additional multivariable analysis was constructed, adding to the model those differences in lipid-related molecules between patients and controls with a ' $p$ ' value lower than 0.20 . Demographic and disease-related data associations with PCSK9 are shown using univariate linear regression. Mediation analysis (27) was performed to assess whether the effect of disease activity on PCSK9 was mediated through other lipid-related molecules. All the analyses used a 5\% two-sided significance level and were performed using SPSS software, version 21 (IBM, Chicago, IL, USA) and STATA software, version 15/SE (Stata Corp., College Station, TX, USA). A $p$ value $<0.05$ was considered statistically significant.

\section{Results}

\section{Demographic, laboratory and disease-related data}

A total of 545 participants, 299 patients with axSpA and 246 controls, with a mean \pm SD age of $52 \pm$ 11 years and $54 \pm 16$ years, respectively, were included in this study. Demographic and disease-related characteristics of the participants are shown in Table 1. Male gender was more frequent in axSpA patients. There were no differences between patients and controls with regard to BMI. However, waist circumference was higher, and the occurrence of diabetes less common in axSpA patients than in controls. In contrast, current smoking frequency, hypertension, and obesity did not reveal any differences between axSpA patients and controls.

The average axSpA disease duration was $13 \pm 9$ years. One hundred and ninety-one (64\%) patients were positive for HLA-B27. Sixteen percent of the patients were categorized as having inactive disease based on the ASDAS-CRP index, while $24 \%, 45 \%$, and $14 \%$ were included in the low, high, and very high disease activity categories, respectively. BASDAI, BASFI and BASMI scores were 4.35 (IQR 2.30-6.00), 3.8 (IQR 1.6-6.3) and 2.4 (IQR 1.0-4.0), respectively. Regarding therapies, $8 \%$ of the axSpA patients were taking prednisone, $61 \%$ were taking non-steroidal anti-inflammatory drugs (NSAIDs), and $28 \%$ and $39 \%$ were, respectively, taking disease-modifying anti-rheumatic drugs (DMARDs) and anti-TNF alpha treatment. Additional disease-related information is shown in Table 1.

\section{Multivariable analysis of the differences in PCSK9 and lipid profiles between SS patients and controls}

In general, the lipid profiles showed that most lipid panel parameters (total cholesterol, HDL- and LDLcholesterol, lipoprotein (a) and apoliprotein A1) were lower in axSpA patients. In contrast, Apo B:Apo A1 and LDL:HDL cholesterol ratios were higher in axSpA patients compared to controls. The mean PCSK9 
serum levels were significantly lower in axSpA patients compared to controls ( $249 \pm 105$ vs. $199 \pm 74$, $\mathrm{ng} / \mathrm{ml}, \mathrm{p}=0.000$ ) when the univariate analysis was performed. When these differences were assessed through multivariable analysis - adjusting for age and sex (including abdominal circumference, smoking and diabetes), total cholesterol, LDL-cholesterol and apolipoprotein A1 - serum levels maintained the same differences, being lower in axSpA patients compared to controls. Similarly, PCSK9 serum levels were significantly downregulated in axSpA patients (beta coef. -40 [95\% $\mathrm{Cl}-56--24] \mathrm{ng} / \mathrm{ml}, 0.000$ ).

Because lipid-related molecules are interrelated (they share metabolic pathways and it is not easy to separate the effect of one from the others), we performed a multivariable analysis adjusting for demographics and $\mathrm{CV}$ risk factors plus all the lipid-related molecules that were found to be different between patients and controls (Model 2 in Table 2). Because of collinearity, lipid molecules derived from a formula were excluded from the regression model (LDL-cholesterol, LDL:HDL ratio, non-HDL cholesterol, apoB:apoA, and atherogenic). HDL-cholesterol (beta coef. 4 [95\% Cl 2-5] mg/dl, $p=0.000$ ), and Apo A1 (beta coef. -13 [95\% $\mathrm{Cl}-16--9] \mathrm{mg} / \mathrm{dl}, \mathrm{p}=0.000$ ) maintained their differences between patients and controls. Remarkably, PCSK9 (beta coef. -44 [95\% Cl -60- -27] \% mg/dl, $p=0.000$ ) conserved its downregulation in axSpA patients after adjustment for other lipid profile-related molecules.

Association of demographic- and disease-specific factors and carotid assessment data with PCSK9 in axSpA patients and controls

Traditional CV risk factors, including BMI and waist circumference, were not related to PCSK9 in patients. In controls, only the presence of hypertension was positively associated with PCSK9 serum levels. Interestingly, age and male sex were respectively positive and negatively related with PCSK9 in patients but not in controls. Concerning lipid profile, some correlations were found between lipid-related molecules and PCSK9. In this regard, triglycerides were positively associated with PCSK9 in both patients and controls; total cholesterol and apolipoprotein A1 serum levels were associated with PCSK9 in patients, but not in controls; and lipoprotein (a) was related to PCSK9 only in controls. Remarkably, statins intake was positively associated with PCSK9 serum levels in patients and controls (Table 3 ).

Regarding disease-related data, disease duration ( $\log$ beta coef. 10 [0-20], $\mathrm{p}=0.043$ ), as well as ASDASCRP (12 [95\% Cl 4-20], $\mathrm{p}=0.004)$ and BASFI ( $\log$ beta coef. 12 [95\% Cl 0-25], $\mathrm{p}=0.049)$ scores, were positively related to PCSK9. Moreover, patients included in the very high disease activity ASDAS-CRP category showed higher serum levels of PCSK9 compared to those in the remission category (32 [95\% Cl $2-63], p=0.038)$. Remarkably, while patients on current prednisone showed higher serum levels of PCSK9 $(55[95 \% \mathrm{Cl} 24-8]) \mathrm{ng} / \mathrm{ml}, \mathrm{p}=0.001)$, patients under anti-TNF alpha therapies exhibited lower levels (beta coef. -26 [95\% Cl-43- -9], $p=0.003$ ).

In assessing the relation of carotid ultrasound results with PCSK9 levels in axSpA patients we observed that CIMT was not related to PCSK9. Although axSpA patients with carotid plaques were associated with higher levels of PCSK9 (beta coef. 27 [95\% Cl 9-45], $p=0.003$ ) in the univariate analysis, this relation was lost after adjustment for confounding factors. The differences between patients with and without carotid plaques are shown in Supplementary Table 1.

Page 6/20 


\section{ASDAS-CRP score relation with lipid profile and PCSK9 serum levels}

ASDAS-CRP score was associated with several lipid profile-related molecules in a univariate fashion. With respect to this, significant univariate correlations were found between ASDAS-CRP and total cholesterol, LDL-cholesterol, LDL: HDL cholesterol, non-HDL cholesterol, lipoprotein (a), atherogenic index, and PCSK9 serum levels (Table 4). When these analyses were performed by fully adjusted models that took into account age, sex, and the rest of the lipid profile molecules also related to ASDAS-CRP, some significant associations were maintained. In this regard, ASDAS-CRP was found to be independently and significantly related to total cholesterol (beta coef. $4[95 \% \mathrm{Cl} 0-8] \mathrm{mg} / \mathrm{dl}, \mathrm{p}=0.027$ ), apolipoprotein A1 (beta coef. -3 [95\% Cl -5- -0] mg/dl, $p=0.019$ ), and PCSK9 (beta coef. $10[95 \% \mathrm{Cl} 1-18] \mathrm{ng} / \mathrm{dl}, \mathrm{p}=0.023$ ). Additionally, mediation analysis disclosed that the effect of ASDAS-CRP on PCSK9 was not mediated by any of the other two cholesterol molecules (cholesterol and apolipoprotein A1) of the previous model. Similarly, neither the effects of ASDAS-CRP on cholesterol nor apolipoprotein A1 were mediated by PCSK9.

\section{Discussion}

In addition to confirming that features of inflammatory dyslipidemia are present in patients with axSpA, in the present study we assessed for the first time PCSK9 in these patients. Although PCSK9 is downregulated in axSpA, a link between disease activity and PCSK9 levels was found.

Previous reports revealed a disrupted lipid profile in axSpA. However, most studies focused on the prevalence of hyperlipidemia in patients with axSpA and only a few compared a full lipid panel between patients and controls assessed in real-life clinical practice. In this regard, studies derived from the ASASCOMOSPA cohort (28-30) defined the presence of dyslipidemia based on the presence of hypercholesterolemia or cholesterol-lowering therapy or on an LDL cholesterol level above target according to country recommendations. However, as neither a complete lipid profile was not assessed nor was a comparison with controls performed, no association analyses were carried out in a multivariate way. Interestingly, in a recent report on the CARMA project (a 10-year prospective cohort study designed to determine the $\mathrm{CV}$ mortality risk in patients with chronic inflammatory rheumatic diseases) (31), patients with axSpA were more likely to have hyperlipoproteinemia (a) than controls after adjusting for age and sex. This association was not found in our cohort.

Lipid profile differences between patients and controls found in our report are in accordance with the 'lipid paradox' (32) described in other inflammatory diseases such as rheumatoid arthritis $(17,33,34)$ or systemic lupus erythematosus (35). This means that individuals with untreated inflammatory diseases or those with these conditions who have high disease activity exhibit lower levels of total cholesterol and LDL cholesterol, and it is believed that this may be due to the lipid-lowering effects of systemic inflammation. The large sample size assessed in the present study allowed us to perform a multivariate 
analysis. For this reason, we believe that our findings regarding lipid profile modifications in axSpA are robust enough to be considered conclusive.

The downregulation of PCSK9 in axSpA patients found in our study is in agreement with a previous report of our group that showed similar findings in in patients with rheumatoid arthritis (17). PCSK9 serum concentrations were lower in axSpA and rheumatoid arthritis patients than in controls. However, the exact mechanisms of PCSK9 downregulation are not yet understood. One explanation could be that PSCK9 levels are downregulated as a compensatory mechanism in the context of heightened cardiovascular disease risk to reduce the accelerated atherosclerosis of these diseases. On the other hand, although higher levels of plasma PCSK9 have been independently associated with major systemic inflammatory markers in patients with acute coronary syndrome and coronary artery disease (36), it is possible that the presence of a persistently elevated chronic proinflammatory state in patients with chronic inflammatory disease would promote PCSK9 downregulation. This would be in agreement with the increased metabolic clearance of total cholesterol and LDL cholesterol observed in these conditions.

Although the effect of glucocorticoids over PCSK9 had not previously been explored, in our study we observed that PCSK9 was related to prednisone intake, being higher in patients treated with glucocorticoids. Therefore, we cannot exclude the possibility that some deleterious effects of glucocorticoids on cardiovascular disease and dyslipidemia might be mediated by this molecule. In contrast, anti-TNF therapies showed a negative association with PCSK9. This result may be of potential interest since the effect of anti-TNF therapy on PCSK9 has not previously been studied. This finding was not observed in a series of 27 patients with rheumatoid arthritis treated with tocilizumab during one year (37). However, in this series, variations in LDL cholesterol over the year of study correlated directly with changes in PCSK9 serum concentration.

In our series, statin use was associated with higher PCSK9 serum levels in patients and controls. This upregulation effect has been previously described in a recent meta-analysis in which statin therapy was shown to increase plasma PCSK9 concentrations, an effect that has been correlated to the magnitude of reduction that statins exert over plasma LDL cholesterol (38).

In our report, PCSK9 was associated with carotid plaques, but not with the cIMT. However, the relation between carotid plaques and PCSK9 was lost after multivariable analysis. It is well established that carotid plaque and cIMT are biologically and genetically distinct entities, representing different phenotypes of atherosclerosis (39). cIMT is thought to represent a mainly hypertensive medial hypertrophy, while carotid plaque is more strongly associated with traditional risk factors and coronary artery disease than is cIMT. Although extensive evidence suggests that there is a significant correlation between PCSK9 levels and future CV risk, scarce data exist about the relationship between PCSK9 levels and vascular biomarkers such as carotid plaque. We believe that the fact that PCSK9 was associated with carotid plaque, though univariately, points to the validity of this molecule as a potential future target in the treatment of atherosclerosis in axSpA patients. 
PCSK9 was downregulated in axSpA patients. Nevertheless, it was positively related to some diseasespecific factors such as disease duration and ASDAS-CRP and BASFI scores. As previously discussed, we believe that although PCSK9 may be reduced due to the presence of a chronic inflammatory state, the absolute levels of this molecule indicate an increased risk of CV disease in the subgroup of patients with more severe disease. With respect to this, ASDAS-CRP was positively associated with total cholesterol, LDL cholesterol, LDL: HDL ratio, non-HDL cholesterol, lipoprotein (a) and atherogenic index in the univariate assessment. Interestingly, the relationships of ASDAS-CRP with total cholesterol, apolipoprotein A1 and PCSK9 remained significant in the multivariate regression analysis. Therefore, these three molecules were related to ASDAS-CRP regardless of other changes that the disease activity might exert over the lipid profile. Additional analysis showed that the effect of disease activity on PCSK9 was not mediated by the influence that the disease activity could exert over cholesterol or apolipoprotein A1. Therefore, while disease activity seems to affect PCSK9, changes in this molecule are not the consequence of other modifications in the lipid panel produced by the disease.

We acknowledge the limitation that controls were not sex-matched. This was because we were preferentially interested in matching for statins intake due to its potential effect over lipid patterns and the effect on PCSK9 serum levels. On the other hand, it is important to highlight that all the analyses in our study were performed by adjusting for important confounders such as age, sex and lipid profile. Nevertheless, identical results have been found irrespective of matching or not matching when multivariable regression analysis was applied in epidemiological cross-sectional or case-control studies (40). We believe, therefore, that the multivariable analysis performed in the present study was capable of handling confounding situations in our analysis regarding sex non-matched controls.

\section{Conclusion}

In conclusion, the fact that PCSK9 is downregulated but positively related to disease activity in patients with axSpA reveals a new mechanism that could explain the presence of increased atherosclerotic burden in patients with axSpA. Further studies are needed to study the role of PCSK9 in other inflammatory diseases.

\section{Abbreviations}

ASDAS: Ankylosing Spondylitis Disease Activity Score; ASAS: Assessment of SpondyloArthritis International Society; axSpA: axial spondyloarthritis; BASDAl: Bath Ankylosing Spondylitis Disease Activity Index; BASF: Bath Ankylosing Spondylitis Functional Index; BASMI: Bath Ankylosing Spondylitis Metrology Index; BMI: body mass index; CIMT: Carotid intima-media thickness CRP: C-reactive protein; CV: cardiovascular; DMARDs: disease-modifying anti-rheumatic drugs; HDL: high-density lipoprotein; LDL: low-density lipoprotein; MASES: Maastricht Ankylosing Spondylitis Enthesitis Score; NSAIDs: nonsteroidal anti-inflammatory drugs; PCSK9: Proprotein convertase subtilisin/kexin type 9.

\section{Declarations}




\section{Acknowledgments}

We thank the Sociedad Española de Reumatología for its assistance in the English-language review of this manuscript.

\section{Authors' contributions}

IFA and MAGG designed and performed research, analyzed data, and wrote the manuscript. IFA, CRL and JCQA acquired the patient samples. LAR, AVG, AGD and JAGD analyzed the blood samples and performed the experiments. AJS helped interpreting data and assisted in preparing the manuscript. All authors read and approved the final manuscript.

\section{Funding}

This work was supported by a grant to I.F-A. from the Spanish Ministry of Health, Subdirección General de Evaluación y Fomento de la Investigación, Plan Estatal de Investigación Científica y Técnica y de Innovación 2013-2016 and by Fondo Europeo de Desarrollo Regional - FEDER - (Fondo de Investigaciones Sanitarias, FIS PI14/00394, PI17/00083).

Prof. González-Gay research is supported by the Instituto de Salud Carlos III (ISCIII) (Fondo de Investigación Sanitaria grants PI06/0024, PI09/00748, PI12/00060, PI15/00525, PI18/00043) and the ISCIII RETICS programs (RD12/0009 and RD16/0012).

\section{Availability of data and materials}

Data are available from the corresponding author on a reasonable request.

\section{Ethics approval and consent to participate}

This study was approved by the institutional review board of the Hospital Universitario de Canarias y Hospital Dr. Negrín de Las Palmas de Gran Canaria (IRB approval number 2016/64) to be in accordance with the ethical standards and with the Helsinki Declaration of 1975, as revised in 2013.

\section{Consent for publication}

Not applicable. 


\section{Competing Interests}

The Authors declare that there is no conflict of interest. Nevertheless, Professor MA Gonzalez-Gay and Dr. Iván Ferraz Amaro would like to acknowledge that they received grants/research supports from Abbott, MSD, Jansen and Roche, and received consultation fees from company-sponsored speakers bureaus associated with Abbott, Pfizer, Roche, Sanofi, Celgene and MSD.

\section{References}

1. Rueda-Gotor J, Corrales A, Blanco R, Fuentevilla P, Portilla V, Expósito R, et al. Atherosclerotic disease in axial spondyloarthritis: increased frequency of carotid plaques. Clin Exp Rheumatol 33:315-20.

2. Rueda-Gotor J, Llorca J, Corrales A, Parra JA, Portilla V, Genre F, et al. Cardiovascular risk stratification in axial spondyloarthritis: carotid ultrasound is more sensitive than coronary artery calcification score to detect high-cardiovascular risk axial spondyloarthritis patients. Clin Exp Rheumatol 36:73-80.

3. Szabo SM, Levy AR, Rao SR, Kirbach SE, Lacaille D, Cifaldi M, et al. Increased risk of cardiovascular and cerebrovascular diseases in individuals with ankylosing spondylitis: a population-based study. Arthritis Rheum. 2011;63:3294-304.

4. Moltó A, Etcheto A, van der Heijde D, Landewé R, van den Bosch F, Bautista Molano W, et al.

Prevalence of comorbidities and evaluation of their screening in spondyloarthritis: results of the international cross-sectional ASAS-COMOSPA study. Ann Rheum Dis. 2016;75:1016-23.

5. Han C, Robinson DW, Hackett MV, Paramore LC, Fraeman KH, Bala MV. Cardiovascular disease and risk factors in patients with rheumatoid arthritis, psoriatic arthritis, and ankylosing spondylitis. $J$ Rheumatol. 2006;33:2167-72.

6. Peters MJ, van der Horst-Bruinsma IE, Dijkmans BA, Nurmohamed MT. Cardiovascular risk profile of patients with spondylarthropathies, particularly ankylosing spondylitis and psoriatic arthritis. Semin Arthritis Rheum. 2004;34:585-92.

7. Divecha H, Sattar N, Rumley A, Cherry L, Lowe GDO, Sturrock R. Cardiovascular risk parameters in men with ankylosing spondylitis in comparison with non-inflammatory control subjects: relevance of systemic inflammation. Clin Sci (Lond). 2005;109:171-6.

8. Van Halm VP, Van Denderen JC, Peters MJL, Twisk JWR, Van Der Paardt M, Van Der Horst-Bruinsma IE, et al. Increased disease activity is associated with a deteriorated lipid profile in patients with ankylosing spondylitis. Ann Rheum Dis Ann Rheum Dis. 2006;65:1473-7.

9. Park SW, Moon Y-A, Horton JD. Post-transcriptional regulation of low density lipoprotein receptor protein by proprotein convertase subtilisin/kexin type 9a in mouse liver. $\mathrm{J}$ Biol Chem. 2004;279:50630-8. 
10. Zhang D-W, Lagace TA, Garuti R, Zhao Z, McDonald M, Horton JD, et al. Binding of proprotein convertase subtilisin/kexin type 9 to epidermal growth factor-like repeat A of low density lipoprotein receptor decreases receptor recycling and increases degradation. J Biol Chem. 2007;282:18602-12.

11. Lee CJ, Lee Y-H, Park SW, Kim KJ, Park S, Youn J-C, et al. Association of serum proprotein convertase subtilisin/kexin type 9 with carotid intima media thickness in hypertensive subjects. Metabolism. 2013;62:845-50.

12. Cohen JC, Boerwinkle E, Mosley TH, Hobbs HH. Sequence variations in PCSK9, low LDL, and protection against coronary heart disease. N Engl J Med. 2006;354:1264-72.

13. Tibolla G, Norata GD, Artali R, Meneghetti F, Catapano AL. Proprotein convertase subtilisin/kexin type 9 (PCSK9): From structure-function relation to therapeutic inhibition. Nutr Metab Cardiovasc Dis. 2011;21:835-43.

14. Roth EM, McKenney JM, Hanotin C, Asset G, Stein EA. Atorvastatin with or without an Antibody to PCSK9 in Primary Hypercholesterolemia. N Engl J Med. 2012;367:1891-900.

15. Sabatine MS, Giugliano RP, Wiviott SD, Raal FJ, Blom DJ, Robinson J, et al. Efficacy and Safety of Evolocumab in Reducing Lipids and Cardiovascular Events. N Engl J Med. 2015;372:1500-9.

16. Robinson JG, Farnier M, Krempf M, Bergeron J, Luc G, Averna M, et al. Efficacy and Safety of Alirocumab in Reducing Lipids and Cardiovascular Events. N Engl J Med. 2015;372:1489-99.

17. Ferraz-Amaro I, López-Mejías R, Ubilla B, Genre F, Tejera-Segura B, de Vera-González AM, et al. Proprotein convertase subtilisin/kexin type 9 in rheumatoid arthritis. Clin Exp Rheumatol 34:1013-9.

18. Rudwaleit M, van der Heijde D, Landewé R, Listing J, Akkoc N, Brandt J, et al. The development of Assessment of SpondyloArthritis international Society classification criteria for axial spondyloarthritis (part II): validation and final selection. Ann Rheum Dis. 2009;68:777-83.

19. Garrett S, Jenkinson T, Kennedy LG, Whitelock H, Gaisford P, Calin A. A new approach to defining disease status in ankylosing spondylitis: the Bath Ankylosing Spondylitis Disease Activity Index. J Rheumatol. 1994;21:2286-91.

20. Lukas C, Landewé R, Sieper J, Dougados M, Davis J, Braun J, et al. Development of an ASASendorsed disease activity score (ASDAS) in patients with ankylosing spondylitis. Ann Rheum Dis. 2009;68:18-24.

21. Calin A, Garrett S, Whitelock H, Kennedy LG, O’Hea J, Mallorie P, et al. A new approach to defining functional ability in ankylosing spondylitis: the development of the Bath Ankylosing Spondylitis Functional Index. J Rheumatol. 1994;21:2281-5.

22. Jenkinson TR, Mallorie PA, Whitelock HC, Kennedy LG, Garrett SL, Calin A. Defining spinal mobility in ankylosing spondylitis (AS). The Bath AS Metrology Index. J Rheumatol. 1994;21:1694-8.

23. Heuft-Dorenbosch L, Spoorenberg A, van Tubergen A, Landewé R, van ver Tempel $H$, Mielants $H$, et al. Assessment of enthesitis in ankylosing spondylitis. Ann Rheum Dis. 2003;62:127-32.

24. Corrales A, González-Juanatey C, Peiró ME, Blanco R, Llorca J, González-Gay MA. Carotid ultrasound is useful for the cardiovascular risk stratification of patients with rheumatoid arthritis: results of a population-based study. Ann Rheum Dis. 2014;73:722-7. 
25. Corrales A, Parra JA, González-Juanatey C, Rueda-Gotor J, Blanco R, Llorca J, et al. Cardiovascular risk stratification in rheumatic diseases: carotid ultrasound is more sensitive than Coronary Artery Calcification Score to detect subclinical atherosclerosis in patients with rheumatoid arthritis. Ann Rheum Dis. 2013;72:1764-70.

26. Touboul P-J, Hennerici MG, Meairs S, Adams H, Amarenco P, Bornstein N, et al. Mannheim carotid intima-media thickness consensus (2004-2006). An update on behalf of the Advisory Board of the 3rd and 4th Watching the Risk Symposium, 13th and 15th European Stroke Conferences, Mannheim, Germany, 2004, and Brussels, Belgium, 2006. Cerebrovasc Dis 2007;23:75-80.

27. Baron RM, Kenny DA. The moderator-mediator variable distinction in social psychological research: Conceptual, strategic, and statistical considerations. J Pers Soc Psychol American Psychological Association (APA); 1986;51:1173-82.

28. Bautista-Molano W, Landewé R, Burgos-Vargas R, Maldonado-Cocco J, Moltó A, Van Den Bosch F, et al. Prevalence of comorbidities and risk factors for comorbidities in patients with spondyloarthritis in Latin america: A comparative study with the general population and data from the ASAS-COMOSPA study. J Rheumatol Journal of Rheumatology. 2018;45:206-12.

29. Moltó A, Etcheto A, Van Der Heijde D, Landewé R, Van Den Bosch F, Bautista Molano W, et al. Prevalence of comorbidities and evaluation of their screening in spondyloarthritis: Results of the international cross-sectional ASAS-COMOSPA study. Ann Rheum Dis BMJ Publishing Group. 2016;75:1016-23.

30. López-Medina C, Jiménez-Gómez Y, Moltó A, Schiotis RE, Marzo-Ortega H, van Gaalen FA, et al. Cardiovascular risk factors in patients with spondyloarthritis from Northern European and Mediterranean countries: An ancillary study of the ASAS-COMOSPA project. Jt Bone Spine Elsevier Masson SAS. 2018;85:447-53.

31. García-Gómez C, Martín-Martínez MA, Fernández-Carballido C, Castañeda S, González-Juanatey C, Sanchez-Alonso F, et al. Hyperlipoproteinaemia(a) in patients with spondyloarthritis: results of the Cardiovascular in Rheumatology (CARMA) project. Clin Exp Rheumatol 37:774-82.

32. González-Gay MA, González-Juanatey C. Inflammation and lipid profile in rheumatoid arthritis: Bridging an apparent paradox. Annals of the Rheumatic Diseases BMJ Publishing Group; 2014. p. 1281-4.

33. Tejera-Segura B, Macía-Díaz M, Machado JD, de Vera-González A, García-Dopico JA, Olmos JM, et al. HDL cholesterol efflux capacity in rheumatoid arthritis patients: contributing factors and relationship with subclinical atherosclerosis. Arthritis Res Ther. 2017;19:113.

34. Ferraz-Amaro I, González-Gay MA, García-Dopico JA, Díaz-González F. Cholesteryl ester transfer protein in patients with rheumatoid arthritis. J Rheumatol. 2013;40:1040-7.

35. Szabó MZ, Szodoray P, Kiss E. Dyslipidemia in systemic lupus erythematosus. Immunol Res. 2017;65:543-50.

36. Momtazi-Borojeni AA, Sabouri-Rad S, Gotto AM, Pirro M, Banach M, Awan Z, et al. PCSK9 and inflammation: A review of experimental and clinical evidence. European Heart Journal - 
Cardiovascular Pharmacotherapy Oxford University Press; 2019. p. 237 - 45.

37. Ferraz-Amaro I, Hernández-Hernández MV, Tejera-Segura B, Delgado-Frías E, Macía-Díaz M, Machado JD, et al. Effect of IL-6 Receptor Blockade on Proprotein Convertase Subtilisin/Kexin Type-9 and Cholesterol Efflux Capacity in Rheumatoid Arthritis Patients. Horm Metab Res. 2019;51:200-9.

38. Sahebkar A, Simental-Mendía LE, Guerrero-Romero F, Golledge J, Watts GF. Effect of statin therapy on plasma proprotein convertase subtilisin kexin 9 (PCSK9) concentrations: A systematic review and meta-analysis of clinical trials. Diabetes, Obes Metab Blackwell Publishing Ltd; 2015;17:1042-55.

39. Spence JD. Measurement of intima-media thickness vs. carotid plaque: uses in patient care, genetic research and evaluation of new therapies. Int J Stroke. 2006;1:216-21.

40. Faresjö T, Faresjö Å. To Match or Not to Match in Epidemiological Studies-Same Outcome but Less Power. Int J Environ Res Public Health. 2010;7:325-32.

\section{Tables}


ble 1. Demographic data of the 299 axSpA patients and 246 controls

\begin{tabular}{|c|c|c|c|}
\hline & Controls $=246$ & axSpA=299 & $p$ \\
\hline Male, n (\%) & $84(34)$ & $209(70)$ & 0.000 \\
\hline Age, years & $54 \pm 16$ & $52 \pm 11$ & 0.054 \\
\hline $\mathrm{BMI}, \mathrm{mg} / \mathrm{cm} 2$ & $27 \pm 6$ & $28 \pm 6$ & 0.45 \\
\hline Waist circumference, $\mathrm{cm}$ & $95 \pm 10$ & $98 \pm 14$ & 0.019 \\
\hline \multicolumn{4}{|l|}{$\underline{\text { morbidity }}$} \\
\hline Hypertension, n (\%) & $92(37)$ & $110(37)$ & 0.88 \\
\hline Current smoking, n (\%) & 47 (19) & $75(25)$ & 0.096 \\
\hline Diabetes, n (\%) & $42(17)$ & $28(9)$ & 0.007 \\
\hline Obesity, n (\%) & $67(27)$ & $81(27)$ & 0.97 \\
\hline Statins, n (\%) & $66(27)$ & $80(27)$ & 0.99 \\
\hline \multicolumn{4}{|l|}{ SpA-related data } \\
\hline $\mathrm{CRP}, \mathrm{mg} / \mathrm{l}$ & $2.03(1.08-4.61)$ & $2.60(0.97-5.65)$ & 0.007 \\
\hline Disease duration, years & & $13 \pm 9$ & \\
\hline HLA-B27, n (\%) & & $191(64)$ & \\
\hline Family history of spondyloarthritis, n (\%) & & $43(14)$ & \\
\hline ASDAS-ESR & & $2.1(1.4-2.9)$ & \\
\hline ASDAS-CRP & & $2.3(1.7-3.1)$ & \\
\hline Inactive disease, $\mathrm{n}(\%)$ & & $51(18)$ & \\
\hline Low disease activity, n (\%) & & $70(24)$ & \\
\hline High disease activity, n (\%) & & $130(45)$ & \\
\hline Very high disease activity, n (\%) & & $40(14)$ & \\
\hline BASDAI, total score & & $4.35(2.30-6.00)$ & \\
\hline BASDAI $>4, \mathrm{n}(\%)$ & & 168 & \\
\hline \multicolumn{4}{|l|}{ BASDAI > 4 and CRP > $5 \mathrm{mg} / \mathrm{dl}, \mathrm{n}(\%)$} \\
\hline BASFI, total score & & $3.8(1.6-6.3)$ & \\
\hline BASMI, total score & & $2.4(1.0-4.0)$ & \\
\hline MASES, total score & & $0(0-2)$ & \\
\hline Sacroiliitis on MRI, n (\%) & & $50(17)$ & \\
\hline Peripheral spondylitis symptoms, n (\%) & & $126(42)$ & \\
\hline Hip involvement, n (\%) & & $63(21)$ & \\
\hline Syndesmophytes, n (\%) & & $124(41)$ & \\
\hline Enthesitis, n (\%) & & $94(31)$ & \\
\hline Dactylitis, n (\%) & & $19(6)$ & \\
\hline \multicolumn{4}{|l|}{ Extraarticular manifestations, n (\%) } \\
\hline Uveitis, n (\%) & & $71(24)$ & \\
\hline Psoriasis, n (\%) & & $20(7)$ & \\
\hline Inflammatory bowel disease, $\mathrm{n}(\%)$ & & $23(8)$ & \\
\hline Current NSAID, n (\%) & & $181(61)$ & \\
\hline Current prednisone, n (\%) & & $23(8)$ & \\
\hline DMARDs, n (\%) & & $83(28)$ & \\
\hline Methotrexate, n (\%) & & $49(16)$ & \\
\hline Sulfasalazine, n (\%) & & $27(9)$ & \\
\hline anti-TNF, n (\%) & & $118(39)$ & \\
\hline
\end{tabular}

ta represent means \pm SD or median (IQR) when data were not normally distributed. 
II: body mass index; LDL: low-density lipoprotein; HDL: high-density lipoprotein; CRP: C reactive protein.

DAS-CRP (Ankylosing Spondylitis Disease Activity Score) categories were defined as: disease activity

1.3 inactivity; $\geq 1.3$ to $<2.1$ low disease activity; $\geq 2.1$ to $<3.5$ high disease activity; $\geq 3.5$ very high.

iAID: Nonsteroidal anti-inflammatory drugs; DMARD: disease-modifying antirheumatic drug.

F: tumor necrosis factor; Obesity: BMI $>30 \mathrm{~kg} / \mathrm{m} 2$.

SFI: Bath Ankylosing Spondylitis Functional Index; BASMI Bath Ankylosing Spondylitis Metrology Index.

SDAI: Bath Ankylosing Spondylitis Disease Activity Index; MASES: Maastricht Ankylosing Spondylitis Enthesitis Score.

mificant ' $p$ ' values are depicted in bold. 
ble 2. Multivariable analysis of the differences in lipid profile and PCSK9 serum levels between axSpA patients and ntrols

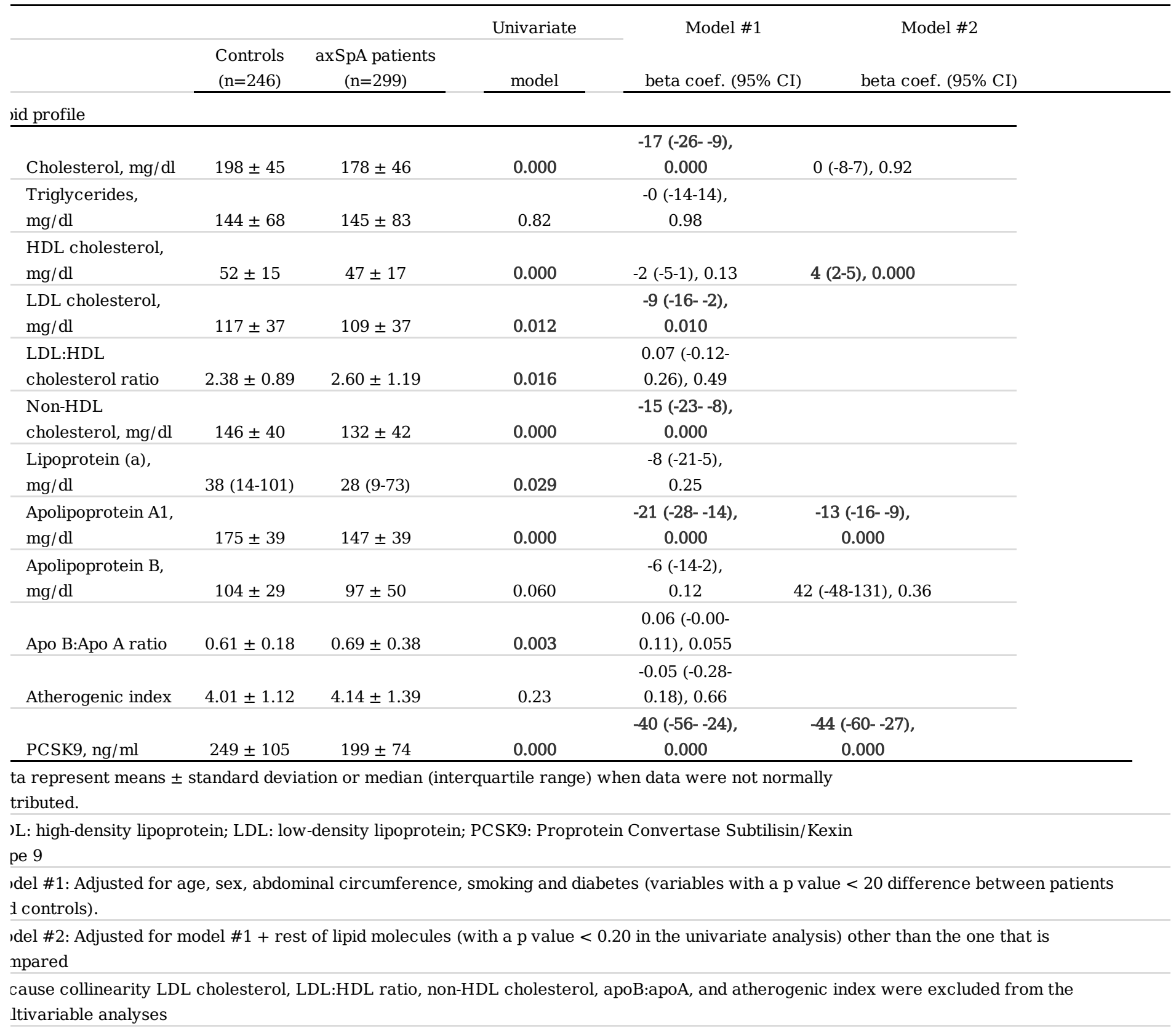

in model 2. 
PCSK9 $\mathrm{ng} / \mathrm{ml}$, beta coef. (CI95\%), p

\begin{tabular}{|c|c|c|}
\hline & Controls $(n=246)$ & axSpA patients $(\mathrm{n}=299)$ \\
\hline Male & $-16(-45-12), 0.25$ & $-25(-44--7), 0.007$ \\
\hline Age, years & 0.97 (0.15-1.79), 0.021 & $1(1-2), 0.000$ \\
\hline $\mathrm{BMI}, \mathrm{mg} / \mathrm{cm} 2$ & $0.93(-1.48-3.34), 0.45$ & $-0.02(-1.47-1.44), 0.98$ \\
\hline Waist circumference, $\mathrm{cm}$ & $-0.58(-1.95-0.79), 0.40$ & $0.07(-0.55-0.68), 0.84$ \\
\hline \multicolumn{3}{|l|}{ orbidity } \\
\hline Hypertension & $22(-5-50), 0.11$ & $25(8-42), 0.005$ \\
\hline Current smoking & $-0.07(-34.01-33.89), 0.99$ & $-3(-23-17), 0.75$ \\
\hline Diabetes & $15(-21-50), 0.42$ & $0.91(-28.04-29.87), 0.95$ \\
\hline Obesity & 25 (-4-55), 0.095 & $6(-13-25), 0.56$ \\
\hline Statins & 72( & $55(36-$ \\
\hline \multicolumn{3}{|l|}{ ytical data } \\
\hline $\log \mathrm{CRP}, \mathrm{mg} / \mathrm{l}$ & 16 (4-29), 0.009 & $5(-2-12), 0.13$ \\
\hline Cholesterol, mg/dl & $-0.20(-0.50-0.10), 0.19$ & $0.20(0.01-0.38), 0.036$ \\
\hline Triglycerides, mg/dl & $0.21(0.01-0.40), 0.036$ & $0.14(0.04-0.24), 0.008$ \\
\hline $\mathrm{HDL}, \mathrm{mg} / \mathrm{dl}$ & $-0.84(-1.75-0.07), 0.071$ & $0.42(-0.08-0.92), 0.10$ \\
\hline LDL, mg/dl & $-0.30(-0.66-0.06), 0.10$ & $0.09(-0.16-0.34), 0.48$ \\
\hline LDL:HDL cholesterol ratio & $-4(-19-11), 0.57$ & $-2(-10-6), 0.59$ \\
\hline Non-HDL cholesterol, mg/dl & $-0.13(-0.47-0.20), 0.43$ & $0.17(-0.03-0.37), 0.10$ \\
\hline Lipoprotein (a), mg/dl & $0.20(0.02-0.38), 0.029$ & $0.12(-0.01-0.25), 0.067$ \\
\hline Apolipoprotein A1 & $-0.19(-0.53-0.16), 0.29$ & $0.33(0.12-0.54), 0.002$ \\
\hline Apolipoprotein B & $-0.16(-0.62-0.31), 0.51$ & $0.16(-0.01-0.33), 0.068$ \\
\hline Apo B:Apo A1 ratio & $-9(-82-65), 0.82$ & $4(-18-27), 0.70$ \\
\hline Atherogenic index & $1(-11-13), 0.84$ & 0.64 (-5.48-6.77), 0.84 \\
\hline
\end{tabular}

losing Spondylitis related data

$\log$ Disease duration, years

$10(0-20), 0.043$

HLA-B27

$-19(-40-1), 0.069$

Family history of spondyloarthritis

$-8(-32-16), 0.53$

ASDAS-CRP

12 (4-20), 0.004

\section{Inactive disease \\ Low disease activity \\ High disease activity \\ Very high disease activity}

-11 (-38-16), 0.41

$-2(-27-22), 0.86$

32 (2-63), 0.038

BASDAI, total score

3 (-1-6), 0.11

BASDAI $>4$

(-10-25), 0.39

$\log$ BASFI, total score

$12(0-25), 0.049$

BASMI, total score

2 (-1-6), 0.22

$\log$ MASES, total score

-0.32 (-11.34-10.70), 0.95

Peripheral spondylitis symptoms

$15(-2-32), 0.081$

Enthesitis

7 (-11-26), 0.43

Extraarticular manifestations

Uveitis

-19 (-39-1), 0.058

Psoriasis

0.03 (-34-35), 0.99

Inflammatory bowel disease

$-22(-54-9), 0.16$

Page $18 / 20$ 
DMARDs

0.55 (-19-19), 0.96

Methotrexate 5 (-18-28), 0.68

Sulfasalazine -19 (-48-10), 0.20

anti-TNF

tid ultrasound assessment

cIMT, mm $12(-61-86), 0.74$

Carotid plaque, 27 (9-45), 0.003

coefficients are expressed without decimals except for those with a value $<1$.

body mass index; LDL: low-density lipoprotein; HDL: high-density lipoprotein; CRP: C reactive protein.

AS-CRP (Ankylosing Spondylitis Disease Activity Score) categories were defined as: disease activity < 1.3 inactivity; 3 to $<2.1$ low disease activity; $\geq 2.1$ to $<3.5$ high disease activity; $\geq 3.5$ very high.

ID: Nonsteroidal anti-inflammatory drugs; DMARD: disease-modifying antirheumatic drug.

: tumor necrosis factor; Obesity: BMI $>30 \mathrm{~kg} / \mathrm{m} 2$.

ЭI: Bath Ankylosing Spondylitis Functional Index; BASMI Bath Ankylosing Spondylitis Metrology Index.

JAI: Bath Ankylosing Spondylitis Disease Activity Index; MASES: Maastricht Ankylosing Spondylitis Enthesitis Score.

ficant ' $\mathrm{p}$ ' are depicted in bold. 


\begin{tabular}{|c|c|c|c|c|c|c|}
\hline \multirow[b]{4}{*}{ Cholesterol, mg/dl } & \multicolumn{6}{|c|}{ ASDAS-CRP } \\
\hline & \multirow{2}{*}{\multicolumn{2}{|c|}{ Pearson $r, p$}} & \multirow{3}{*}{$\frac{\text { beta coef. }(95 \% \mathrm{CI}), \mathrm{p}}{4(0-8), 0.027}$} & \multicolumn{3}{|c|}{ Mediation analysis } \\
\hline & & & & \multirow{2}{*}{$\begin{array}{c}\text { Cholesterol } \\
-\end{array}$} & \multirow{2}{*}{$\frac{\text { Apo A1 }}{0.84}$} & \multirow{2}{*}{$\frac{\text { PCSK9 }}{0.94}$} \\
\hline & 0.135 & 0.022 & & & & \\
\hline Triglycerides, mg/dl & 0.104 & 0.080 & $0(-8-7), 0.93$ & & & \\
\hline HDL cholesterol, mg/dl & 0.014 & 0.82 & $0(-1-2), 0.42$ & & & \\
\hline LDL cholesterol, mg/dl & 0.121 & 0.041 & & & & \\
\hline LDL:HDL cholesterol ratio & 0.149 & 0.011 & & & & \\
\hline Non-HDL cholesterol, mg/dl & 0.141 & 0.017 & & & & \\
\hline Lipoprotein (a), mg/dl & 0.122 & 0.040 & $5(-2-13), 0.17$ & & & \\
\hline Apolipoprotein A1, mg/dl & -0.020 & 0.74 & $-3(-5--0), 0.019$ & 0.19 & - & 0.31 \\
\hline Apolipoprotein B, mg/dl & 0.063 & 0.29 & 20 (-55-95), 0.60 & & & \\
\hline Apo B:Apo A ratio & 0.080 & 0.18 & & & & \\
\hline Atherogenic index & 0.148 & 0.012 & & & & \\
\hline PCSK9, ng/ml & 0.170 & 0.004 & 10 (1-18), 0.023 & 0.57 & 0.97 & - \\
\hline
\end{tabular}

HDL: high-density lipoprotein; LDL: low-density lipoprotein; PCSK9: Proprotein Convertase Subtilisin/Kexin Type 9

Beta coef. results are adjusted for age, sex, and all lipid profile molecules other than the one that is compared

Because collinearity LDL cholesterol, LDL:HDL ratio, non-HDL cholesterol, apoB:apoA, and atherogenic index were excluded from the multivariable analysis.

ASDAS-CRP is considered the independent variable in the regression analysis

\section{Supplementary Files}

This is a list of supplementary files associated with this preprint. Click to download.

- SupplementaryTable1.docx 